\title{
CONVERGENCE AND INTEGRABILITY OF DOUBLE TRIGONOMETRIC SERIES WITH COEFFICIENTS OF BOUNDED VARIATION
}

\author{
FERENC MÓRICZ
}

(Communicated by Richard R. Goldberg)

Abstract. We prove that if $c(j, k) \rightarrow 0$ as $\max (|j|,|k|) \rightarrow \infty$ and

$$
\sum_{j=-\infty}^{\infty} \sum_{k=-\infty}^{\infty}\left|\Delta_{11} c(j, k)\right|<\infty
$$

then the series $\sum_{j=-\infty}^{\infty} \sum_{k=-\infty}^{\infty} c(j, k) e^{i(j x+k y)}$ converges both pointwise for every $(x, y) \in(T \backslash\{0\})^{2}$ and in the $L^{p}\left(T^{2}\right)$-metric for $0<p<1$, where $T$ is the one-dimensional torus. Both convergence statements remain valid for the three conjugate series under these same coefficient conditions.

1. Introduction. We will consider double trigonometric series of the form

$$
\sum_{j=-\infty}^{\infty} \sum_{k=-\infty}^{\infty} c(j, k) e^{i(j x+k y)}
$$

where $\{c(j, k):-\infty<j, k<\infty\}$ is a null sequence of complex numbers. Here and in the sequel,

$$
(x, y) \in T^{2}:=\left\{(x, y) \in R^{2}: 0 \leq x, y<2 \pi\right\}
$$

the two-dimensional torus, whereas $T:=\{x \in R: 0 \leq x<2 \pi\}$.

The pointwise convergence of series (1.1) is usually defined in Pringsheim's sense (see, e.g. [5, Vol. 2, Chapter 17]). This means that we form the symmetric partial sums

$$
s_{M N}(x, y):=\sum_{j=-M}^{M} \sum_{k=-N}^{N} c(j, k) e^{i(j x+k y)} \quad(M, N \geq 0)
$$

and then let both $M$ and $N$ tend to $\infty$, independently of one another, and assign the limit $f(x, y)$ (if it exists) to series (1.1) as its sum.

Following Hardy [1], series (1.1) is said to be regularly convergent if

(i) it converges in Pringsheim's sense, and

(ii) the single series

$$
\sum_{j=-\infty}^{\infty} c(j, k) e^{i(j x+k y)}
$$

Received by the editors December 19, 1986.

1980 Mathematics Subject Classification (1985 Revision). Primary 42A20, 42A32.

Key words and phrases. Double trigonometric series, conjugate series, double sequences of bounded variation, pointwise convergence, convergence in the $L^{p}\left(T^{2}\right)$-metric for $0<p<1$.

This research was completed while the author was a visiting professor at Syracuse University, New York State, in the academic year 1986/87. 
converges for each fixed value of $k$, and the single series

$$
\sum_{k=-\infty}^{\infty} c(j, k) e^{i(j x+k y)}
$$

converges for each fixed value of $j$.

As is known, if (i) and (ii) are satisfied, then the sum $f(x, y)$ of series (1.1) can be computed in the way of iterated summations, too:

$$
\begin{aligned}
f(x, y) & =\sum_{j=-\infty}^{\infty}\left[\sum_{k=-\infty}^{\infty} c(j, k) e^{i(j x+k y)}\right] \\
& =\sum_{k=-\infty}^{\infty}\left[\sum_{j=-\infty}^{\infty} c(j, k) e^{i(j x+k y)}\right] .
\end{aligned}
$$

The notion of regular convergence was rediscovered in [2] where it was defined by the following equivalent condition (and called "convergence in a restricted sense"): the sums

$$
\sum_{M_{1} \leq|j| \leq M_{2}} \sum_{N_{1} \leq|k| \leq N_{2}} c(j, k) e^{i(j x+k y)} \rightarrow 0
$$

as $\max \left(M_{1}, N_{1}\right) \rightarrow \infty$, independently of the choices of $M_{2}$ and $N_{2}$ where $0 \leq$ $M_{1} \leq M_{2}$ and $0 \leq N_{1} \leq N_{2}$.

Now we introduce an even stronger notion of convergence, we may call it strongly regular convergence, which requires that the sums (with $M_{1} \leq M_{2}$ and $N_{1} \leq N_{2}$ )

$$
s(Q ; x, y):=\sum_{j=M_{1}}^{M_{2}} \sum_{k=N_{1}}^{N_{2}} c(j, k) e^{i(j x+k y)} \rightarrow 0
$$

in each of the following limiting cases:

$$
\left\{\begin{array}{l}
\text { (i) } M_{1} \rightarrow \infty \text {, while } M_{2}, N_{1} \text {, and } N_{2} \text { are arbitrary. } \\
\text { (ii) } M_{2} \rightarrow-\infty \text {, while } M_{1}, N_{1} \text {, and } N_{2} \text { are arbitrary. } \\
\text { (iii) } N_{1} \rightarrow \infty \text {, while } N_{2}, M_{1} \text {, and } M_{2} \text { are arbitrary. } \\
\text { (iv) } N_{2} \rightarrow-\infty \text {, while } N_{1}, M_{1} \text {, and } M_{2} \text { are arbitrary. }
\end{array}\right.
$$

Here and in the sequel, $Q$ denotes the set of the lattice points of the plane contained in the rectangle $\left\{(j, k): M_{1} \leq j \leq M_{2}\right.$ and $\left.N_{1} \leq k \leq N_{2}\right\}$.

After these convergence notions, we repeat the definitions of the three conjugate series to (1.1):

$$
\sum_{j=-\infty}^{\infty} \sum_{k=-\infty}^{\infty}(-i \operatorname{sgn} j) c(j, k) e^{i(j x+k y)}
$$

(conjugate with respect to $x$ ),

$$
\sum_{j=-\infty}^{\infty} \sum_{k=-\infty}^{\infty}(i \operatorname{sgn} k) c(j, k) e^{i(j x+k y)}
$$

(conjugate with respect to $y$ ), 


$$
\sum_{j=-\infty}^{\infty} \sum_{k=-\infty}^{\infty}(-i \operatorname{sgn} j)(-i \operatorname{sgn} k) c(j, k) e^{i(j x+k y)}
$$

(conjugate with respect to $x$ and $y$ ).

If series (1.4)-(1.6) converge in Pringsheim's sense, then their sums are denoted by $\tilde{f}^{(1,0)}(x, y), \tilde{f}^{(0,1)}(x, y)$, and $\tilde{f}^{(1,1)}(x, y)$, respectively, and are called the corresponding conjugate functions to $f(x, y)$ (see, e.g., $[3]$ ).

2. Main results. Let $\{c(j, k):-\infty<j, k<\infty\}$ be a double sequence. We remind the reader that its differences are defined by

$$
\begin{aligned}
& \Delta_{10} c(j, k)=c(j, k)-c(j+1, k), \\
& \Delta_{01} c(j, k)=c(j, k)-c(j, k+1), \\
& \Delta_{11} c(j, k)=c(j, k)-c(j+1, k)-c(j, k+1)+c(j+1, k+1),
\end{aligned}
$$

and $\{c(j, k)\}$ is said to be of bcunded variation if

$$
C_{11}:=\sum_{j=-\infty}^{\infty} \sum_{k=-\infty}^{\infty}\left|\Delta_{11} c(j, k)\right|<\infty
$$

We will prove the following convergence statements.

THEOREM. Let $\{c(j, k):-\infty<j, k<\infty\}$ be a double sequence of complex numbers that is of bounded variation and such that

$$
c(j, k) \rightarrow 0 \quad \text { as } \max (|j|,|k|) \rightarrow \infty .
$$

Then series (1.1)

(i) converges pointwise in the strongly regular sense to some function $f(x, y)$ for every $(x, y) \in(T \backslash\{0\})^{2}$;

(ii) converges in the $L^{p}\left(T^{2}\right)$-metric to $f$ for every $0<p<1$ :

$$
\left\|\sum_{j=M_{1}}^{M_{2}} \sum_{k=N_{1}}^{N_{2}} c(j, k) e^{i(j x+k y)}-f(x, y)\right\|_{p} \rightarrow 0
$$

as $M_{2}, N_{2} \rightarrow \infty$ and $M_{1}, N_{1} \rightarrow-\infty$. In particular, we have $f \in L^{p}\left(T^{2}\right)$ for every $0<p<1$.

(iii) Analogous conclusions can be drawn for the conjugate series (1.4)-(1.6) and the corresponding conjugate functions $\tilde{f}^{(1,0)}, \tilde{f}^{(0,1)}, \tilde{f}^{(1,1)}$, respectively.

Here and in the sequel, $\|\cdot\|_{p}$ denotes the $L^{p}\left(T^{2}\right)$-norm defined by

$$
\|g\|_{p}:=\left[\int_{0}^{2 \pi} \int_{0}^{2 \pi}|g(x, y)|^{p} d x d y\right]^{1 / p} .
$$

Our Theorem can be considered the extension of a theorem of Uljanov [4] from single to double trigonometric series. 
3. Auxiliary results. We present two lemmas.

LEMMA 1. If $\{c(j, k)\}$ satisfies conditions (2.1) and (2.2), then for every $k$,

$$
\begin{gathered}
\sum_{j=-\infty}^{\infty}\left|\Delta_{10} c(j, k)\right| \leq C_{11}(<\infty) \\
\sum_{j=-\infty}^{\infty}\left|\Delta_{10} c(j, k)\right| \rightarrow 0 \quad \text { as }|k| \rightarrow \infty
\end{gathered}
$$

$$
\sup _{k} \sum_{|j|>M}\left|\Delta_{10} c(j, k)\right| \rightarrow 0 \quad \text { as } M \rightarrow \infty .
$$

We note that analogous statements are available for the series $\sum_{k=-\infty}^{\infty}\left|\Delta_{01} c(j, k)\right|$ under the same conditions.

ProOF. By (2.2),

$$
\Delta_{10} c\left(j, k_{0}\right)=\sum_{k=k_{0}}^{\infty} \Delta_{11} c(j, k)
$$

whence

$$
\sum_{j=-\infty}^{\infty}\left|\Delta_{10} c\left(j, k_{0}\right)\right| \leq \sum_{j=-\infty}^{\infty} \sum_{k=k_{0}}^{\infty}\left|\Delta_{11} c(j, k)\right|
$$

and (3.1) follows.

Again by (2.2),

$$
\Delta_{10} c\left(j, k_{0}\right)=-\sum_{k=-\infty}^{k_{0}-1} \Delta_{11} c(j, k)
$$

whence

$$
\sum_{j=-\infty}^{\infty}\left|\Delta_{10} c\left(j, k_{0}\right)\right| \leq \sum_{j=-\infty}^{\infty} \sum_{k=-\infty}^{k_{0}-1}\left|\Delta_{11} c(j, k)\right| .
$$

Clearly (3.2) follows from (2.1), (3.4), and (3.5).

Finally, (3.3) is a consequence of (3.2) (applied for large values of $|k|$ ) and (3.1) (applied for small values of $|k|$ ).

LEMMA 2. If $\{c(j, k)\}$ satisfies conditions (2.1) and (2.2), then the sequences

$$
\left\{\begin{aligned}
\text { (i) } & \{(-i \operatorname{sgn} j) c(j, k)\}, \\
\text { (ii) } & \{(-i \operatorname{sgn} k) c(j, k)\}, \\
\text { (iii) } & \{(-i \operatorname{sgn} j)(-i \operatorname{sgn} k) c(j, k)\}
\end{aligned}\right.
$$

also satisfy these same conditions. 
PROOF. It suffices to prove (i), since (ii) is a symmetric counterpart of (i), while (iii) follows from a repeated application of (i) and (ii). In the case of (i), the fulfillment of (2.2) is obvious. Inequality (2.1) follows from Lemma 1:

$$
\begin{aligned}
\sum_{j=-\infty}^{\infty} & \sum_{k=-\infty}^{\infty}\left|\Delta_{11}[(-i \operatorname{sgn} j) c(j, k)]\right| \\
= & \left\{\sum_{j=-\infty}^{-2} \sum_{k=-\infty}^{\infty}+\sum_{j=1}^{\infty} \sum_{k=-\infty}^{\infty}\right\}\left|\Delta_{11} c(j, k)\right| \\
& +\sum_{k=-\infty}^{\infty}\left|\Delta_{01} c(-1, k)\right|+\sum_{k=-\infty}^{\infty}\left|\Delta_{01} c(1, k)\right| .
\end{aligned}
$$

4. Proof of the Theorem. We will use the notation $w(x)=1-e^{-i x}$. Then

$$
|w(x)|=2 \sin \frac{x}{2} \quad \text { for } 0 \leq x<2 \pi
$$

and

$$
w(x) w(y)=1-e^{-i x}-e^{-i y}+e^{i(x+y)}
$$

Part 1: Pointwise convergence. Performing an "Abel transformation-like" rearrangement yields, for any $M_{1} \leq M_{2}$ and $N_{1} \leq N_{2}$,

$$
\begin{aligned}
w(x) w(y) & \sum_{j=M_{1}}^{M_{2}} \sum_{k=N_{1}}^{N_{2}} c(j, k) e^{i(j x+k y)} \\
= & \sum_{j=M_{1}-1}^{M_{2}-1} \sum_{k=N_{1}-1}^{N_{2}-1} \Delta_{11} c(j, k) e^{i(j x+k y)} \\
& +\sum_{j=M_{1}-1}^{M_{2}-1} \Delta_{10} c\left(j, N_{2}\right) e^{i\left(j x+N_{2} y\right)} \\
& -\sum_{j=M_{1}-1}^{M_{2}-1} \Delta_{10} c\left(j, N_{1}-1\right) e^{i\left(j x+\left(N_{1}-1\right) y\right)} \\
& +\sum_{k=N_{1}-1}^{N_{2}-1} \Delta_{01} c\left(M_{2}, k\right) e^{i\left(M_{2} x+k y\right)} \\
& -\sum_{k=N_{1}-1}^{N_{2}-1} \Delta_{01} c\left(M_{1}-1, k\right) e^{i\left(\left(M_{1}-1\right) x+k y\right)} \\
& +c\left(M_{2}, N_{2}\right) e^{i\left(M_{2} x+N_{2} y\right)}
\end{aligned}
$$


Hence, using (4.1) and notation (1.2),

$$
\begin{aligned}
|s(Q ; x, y)| \leq \frac{1}{4 \sin \frac{x}{2} \sin \frac{y}{2}}\{ & \sum_{j=M_{1}-1}^{M_{2}-1} \sum_{k=N_{1}-1}^{N_{2}-1}\left|\Delta_{11} c(j, k)\right| \\
& +\sum_{j=M_{1}-1}^{M_{2}-1}\left[\left|\Delta_{10} c\left(j, N_{2}\right)\right|+\left|\Delta_{10} c\left(j, N_{1}-1\right)\right|\right] \\
& \left.+\sum_{k=N_{1}-1}^{N_{2}-1}\left[\left|\Delta_{01} c\left(M_{2}, k\right)\right|+\left|\Delta_{01} c\left(M_{1}-1, k\right)\right|\right]+\left|c\left(M_{2}, N_{2}\right)\right|\right\} .
\end{aligned}
$$

Making use of Lemma 1, we can see that each term in the braces on the righthand side tends to zero as any one of the limiting cases (i)-(iv) in (1.3) is realized. Thus, the sum $f(x, y)$ of series (1.1) certainly exists for all $0<x, y<2 \pi$.

Part 2: $L^{p}\left(T^{2}\right)$-convergence. It is plain that

$$
\begin{aligned}
|f(x, y)-s(Q ; x, y)| \leq & \left|\sum_{j=-\infty}^{M_{1}-1} \sum_{k=-\infty}^{\infty} c(j, k) e^{i(j x+k y)}\right| \\
& +\left|\sum_{j=M_{2}+1}^{\infty} \sum_{k=-\infty}^{\infty} c(j, k) e^{i(j x+k y)}\right| \\
& +\left|\sum_{j=M_{1}}^{M_{2}} \sum_{k=-\infty}^{N_{1}-1} c(j, k) e^{i(j x+k y)}\right| \\
& +\left|\sum_{j=M_{1}}^{M_{2}} \sum_{k=N_{2}+1}^{\infty} c(j, k) e^{i(j x+k y)}\right| \\
=: & \sum_{1}+\sum_{2}+\sum_{3}+\sum_{4}, \text { say. }
\end{aligned}
$$

Similarly to (4.2), for any $M>M_{2}$ and $N>0$,

$$
\begin{aligned}
w(x) w(y) & \sum_{j=M_{2}+1}^{M} \sum_{k=-N}^{N} c(j, k) e^{i(j x+k y)} \\
= & \sum_{j=M_{2}}^{M-1} \sum_{k=-N-1}^{N-1} \Delta_{11} c(j, k) e^{i(j x+k y)}+\sum_{j=M_{2}}^{M-1} \Delta_{10} c(j, N) e^{i(j x+N y)} \\
& -\sum_{j=M_{2}}^{M-1} \Delta_{10} c(j,-N-1) e^{i(j x-(N+1) y)} \\
& +\sum_{k=-N-1}^{N-1} \Delta_{01} c(M, k) e^{i(M x+k y)} \\
& -\sum_{k=-N-1}^{N-1} \Delta_{01} c\left(M_{2}, k\right) e^{i\left(M_{2} x+k y\right)}+c(M, N) e^{i(M x+N y) .}
\end{aligned}
$$


Letting $M, N \rightarrow \infty$, by Lemma 1 we get that

$$
\begin{aligned}
w(x) & w(y) \sum_{j=M_{2}+1}^{\infty} \sum_{k=-\infty}^{\infty} c(j, k) e^{i(j x+k y)} \\
& =\sum_{j=M_{2}}^{\infty} \sum_{k=-\infty}^{\infty} \Delta_{11} c(j, k) e^{i(j x+k y)}-\sum_{k=-\infty}^{\infty} \Delta_{01} c\left(M_{2}, k\right) e^{i\left(M_{2} x+k y\right)} .
\end{aligned}
$$

Hence

$$
\sum_{2} \leq \frac{1}{|w(x) w(y)|}\left\{\sum_{j=M_{2}+1}^{\infty} \sum_{k=-\infty}^{\infty}\left|\Delta_{11} c(j, k)\right|+\sum_{k=-\infty}^{\infty}\left|\dot{\Delta}_{01} c\left(M_{2}, k\right)\right|\right\} .
$$

By (4.1), for $0<p<1$,

$$
\left\|\frac{1}{w(x) w(y)}\right\|_{p}=\left[\int_{0}^{2 \pi} \int_{0}^{2 \pi} \frac{d x d y}{4^{p} \sin ^{p} \frac{x}{2} \sin ^{p} \frac{y}{2}}\right]^{1 / p}<\infty .
$$

So, by (2.1) and the counterpart of (3.2) (when the roles of $j$ and $k$ are interchanged), we obtain that

$$
\left\|\sum_{2}\right\|_{p} \rightarrow 0 \text { as } M_{2} \rightarrow \infty
$$

In a similar way, we can deduce that

$$
\left\|\sum_{1}\right\|_{p} \rightarrow 0 \text { as } M_{1} \rightarrow-\infty
$$

Estimating $\sum_{4}$, let $N>N_{2}$. Then, following the pattern occurring in (4.2),

$$
\begin{aligned}
w(x) w & (y) \sum_{j=M_{1}}^{M_{2}} \sum_{k=N_{2}+1}^{N} c(j, k) e^{i(j x+k y)} \\
= & \sum_{j=M_{1}-1}^{M_{2}-1} \sum_{k=N_{2}}^{N-1} \Delta_{11} c(j, k) e^{i(j x+k y)} \\
& +\sum_{j=M_{1}-1}^{M_{2}-1} \Delta_{10} c(j, N) e^{i(j x+N y)} \\
& -\sum_{j=M_{1}-1}^{M_{2}-1} \Delta_{10} c\left(j, N_{2}\right) e^{i\left(j x+N_{2} y\right)} \\
& +\sum_{k=N_{2}}^{N-1} \Delta_{01} c\left(M_{2}, k\right) e^{i\left(M_{2} x+k y\right)} \\
& -\sum_{k=N_{2}}^{N-1} \Delta_{01} c\left(M_{1}-1, k\right) e^{i\left(\left(M_{1}-1\right) x+k y\right)}+c\left(M_{2}, N\right) e^{i\left(M_{2} x+N y\right)}
\end{aligned}
$$


Letting $N \rightarrow \infty$, by Lemma 1 ,

$$
\begin{aligned}
w(x) w(y) \sum_{j=M_{1}}^{M_{2}} \sum_{k=N_{2}+1}^{\infty} c(j, k) e^{i(j x+k y)}= & \sum_{j=M_{1}-1}^{M_{2}-1} \sum_{k=N_{2}}^{\infty} \Delta_{11} c(j, k) e^{i(j x+k y)} \\
& -\sum_{j=M_{1}-1}^{M_{2}-1} \Delta_{10} c\left(j, N_{2}\right) e^{i\left(j x+N_{2} y\right)} \\
& +\sum_{k=N_{2}}^{\infty} \Delta_{01} c\left(M_{2}, k\right) e^{i\left(M_{2} x+k y\right)} \\
& -\sum_{k=N_{2}}^{\infty} \Delta_{01} c\left(M_{1}-1, k\right) e^{i\left(\left(M_{1}-1\right) x+k y\right)}
\end{aligned}
$$

Hence

$$
\begin{aligned}
\sum_{4} \leq \frac{1}{|w(x) w(y)|}\left\{\sum_{j=M_{1}-1} \sum_{k=N_{2}}^{M_{2}-1}\left|\Delta_{11} c(j, k)\right|_{\bullet}+\sum_{j=M_{1}-1}^{M_{2}-1}\left|\Delta_{10} c\left(j, N_{2}\right)\right|\right. \\
\left.\quad+\sum_{k=N_{2}}^{\infty}\left|\Delta_{01} c\left(M_{2}, k\right)\right|+\sum_{k=N_{2}}^{\infty}\left|\Delta_{01} c\left(M_{1}-1, k\right)\right|\right\} .
\end{aligned}
$$

By (2.1), (3.2), and its counterpart, we get that

$$
\left\|\sum_{4}\right\|_{p} \rightarrow 0 \text { as } N_{2} \rightarrow \infty
$$

A similar argument gives that

$$
\left\|\sum_{3}\right\|_{p} \rightarrow 0 \text { as } N_{1} \rightarrow-\infty
$$

Combining (4.3), (4.6), (4.7), (4.9), and (4.10) yields (2.3).

Part 3: Convergence of the conjugate series. This immediately follows from (i) and (ii) of the Theorem, via Lemma 2.

\section{REFERENCES}

1. G. H. Hardy, On the convergence of certain multiple series, Proc. Cambridge Philos. Soc. 19 (1916-1919), 86-95.

2. F. Móricz, On the convergence in a restricted sense of multiple series, Anal. Math. 5 (1979), 135-147.

3. F. Móricz and Xianliang Shi, Approximation to continuous functions by Cesàro means of double Fourier series and conjugate series, J. Approx. Theory 49 (1987), 346-377.

4. P. L. Uljanov, Application of A-integration on a class of trigonometric series, Mat. Sb. $35(77)$ (1954), 469-490. (Russian)

5. A. Zygmund, Trigonometric series, Cambridge Univ. Press, New York, 1959.

Bolyai Institute, University of Szeged, ARAdi VéRtanúk TeRe 1, 6720 Szeged, HUNGARY

Current address: Department of Mathematics, University of Tennessee, Knoxville, Tennessee 37996 